\title{
Higher Order Moments and Recurrence Relations of Order Statistics from the Exponentiated Gamma Distribution
}

\author{
A. I. Shawky and R. A. Bakoban ${ }^{*}$ \\ King Abdulaziz University
}

\begin{abstract}
Order statistics arising from exponentiated gamma (EG) distribution are considered. Closed form expressions for the single and double moments of order statistics are derived. Measures of skewness and kurtosis of the probability density function of the $r$ th order statistic for different choices of $r, n$ and $\theta$ are presented. Recurrence relations between single and double moments of $r$ th order statistics are obtained. Single moment generating function (MGF) is derived in closed form. Also, we establish several recurrence relations between single MGF.
\end{abstract}

Keywords. Order statistics; recurrence relations; single moments; double moments; moment generating function; exponentiated gamma distribution.

\section{Introduction}

The subject of order statistics has been in process of development for many years and recently has become increasingly important. Articles relating to this area have appeared in numerous different publications. Many authors have studied order statistics; for example, David (1981), Balakrishnan and Cohen (1991), Arnold et al. (1992) and David and Nagaraja (2003). Moments of order statistics has been discussed by many authors; for example, Balakrishnan and Gupta (1998), Basu and Singh (1998) and Raqab (1998). Further, measures of skewness and kurtosis of the densities of order statistics from Burr type X distribution were computed by Raqab (1998). Moreover,

* Corresponding author 
recurrence relations for order statistics has dealt with many authors; for example, David (1995), Balakrishnan and Sultan (1998), Balakrishnan and Aggarwala (1998) and Balasubramanian and Beg (2004). Also, Raqab (2004) has established some recurrence relations for moment generating function (MGF) of order statistics from generalized exponential distribution.

For the gamma distribution with shape parameter $\alpha=m$ and scale parameter $\beta=1$ i.e. $G(m, 1)$ explicit expressions for the moments of order statistics have been derived by Gupta (1960) for integer values of $m$, and by Krishnaiah and Rizvi (1967) for a general value of $m$; see also Breiter and Krishnaiah (1968). For integral values of $m$; Joshi (1979) has established recurrence relations satisfied by the single moments of order statistics. Young (1971) has also deduced a simple relation between moments of order statistics from the symmetrical inverse multinomial distribution and the order statistics of independent standardized gamma variable with integer parameter $m$.

Let $X_{1}, X_{2}, X_{3}, \ldots, X_{n}$ be a random sample of size $n$ from exponentiated gamma (EG) distribution with probability density function (p.d.f.)

$$
f(x ; \theta)=\theta x e^{-x}\left(1-e^{-x}-x e^{-x}\right)^{\theta-1}, \quad x>0, \quad \theta>0
$$

and the cumulative distribution function (c.d.f.) is given by

$$
F(x ; \theta)=\left(1-e^{-x}-x e^{-x}\right)^{\theta}, \quad x>0, \quad \theta>0
$$

Other statistical properties of this distribution are discussed by Shawky and Bakoban (2008c). Also, Bayesian estimations on the EG distribution studied by Shawky and Bakoban (2008a). Moreover, characterization from EG distribution based on record values deduced by Shawky and Bakoban (2008b). By putting $\theta=1$ in (1), we have a gamma distribution $G(2,1)$.

From (1) and (2), we observe that the characterization differential equation for the EG distribution is

$$
(x+1) f(x)=\theta x\{F(x)\}^{1-L}\left[1-\{F(x)\}^{L}\right], \quad L=\frac{1}{\theta}, x>0,
$$

$L$ is a positive integer number.

In this paper, we derived exact expressions for the single and double moments of order statistics from EG distribution which is presented in section 2. We compute the measures of skewness and kurtosis of the distribution of the $r$ th order statistic in a sample of size $n$ for different choices of $n, r$ 
and $\theta$. In section 3 , recurrence relations for single and double moments of order statistics are derived. MGF for the single moments of order statistics from EG distribution is derived in section 4. Moreover, we establish some recurrence relations for MGF of order statistics from EG distribution in section 4 .

\section{Moments of Order Statistics}

Let $X_{1: n} \leqslant X_{2: n} \leqslant \cdots \leqslant X_{n: n}$ be the order statistics from the EG distribution given in (1). Then the single and double moments of order statistics are given as follows:

\subsection{Single Moments}

The p.d.f. of the $r$ th order statistic $X_{r: n}, r=1,2, \ldots, n$ given by

$$
f_{r: n}(x)=C_{r: n}\{F(x ; \theta)\}^{r-1}\{1-F(x ; \theta)\}^{n-r} f(x ; \theta), \quad x>0,
$$

(see David, 1981) where $f(\cdot)$ and $F(\cdot)$ are given by (1) and (2), respectively, and

$$
C_{r: n}=\frac{n !}{(r-1) !(n-r) !} .
$$

$f_{r: n}(x)$ can be written as

$$
f_{r: n}(x)=\sum_{i=0}^{n-r} d_{i}(n, r) f\{x ; \theta(r+i)\}
$$

where

$$
d_{i}(n, r)=(-1)^{i} n \frac{\left(\begin{array}{c}
n-1 \\
r-1
\end{array}\right)\left(\begin{array}{c}
n-r \\
i
\end{array}\right)}{r+i}
$$

The ath moment of the $r$ th order statistic, $E\left(X_{r: n}^{a}\right)$, denoted by $\alpha_{r: n}^{(a)}, 1 \leqslant$ $r \leqslant n$ and $a=0,1,2, \ldots$, is given by

$$
\alpha_{r: n}^{(a)}=\int_{0}^{\infty} x^{a} f_{r: n}(x ; \theta) d x .
$$

The exact explicit expression for the single moments of order statistics from EG distribution is given by the following theorem: 
Theorem 1 For $1 \leqslant r \leqslant n, a \geqslant 0$ and $\theta$ is a real value, then

$$
\alpha_{r: n}^{(a)}=\theta C_{r: n} \sum_{i=0}^{n-r} \sum_{j=0}^{\infty} \sum_{k=0}^{j}(-1)^{i+j}\left(\begin{array}{c}
n-r \\
i
\end{array}\right)\left(\begin{array}{c}
\theta(r+i)-1 \\
j
\end{array}\right)\left(\begin{array}{l}
j \\
k
\end{array}\right) \frac{\Gamma(a+k+2)}{(j+1)^{a+k+2}}
$$

Proof. From (1), (5) and (7) we get

$$
\alpha_{r: n}^{(a)}=\sum_{i=0}^{n-r} d_{i}(n, r) \theta(r+i) \int_{0}^{\infty} x^{a+1} e^{-x}\left\{1-e^{-x}(x+1)\right\}^{\theta(r+i)-1} d x .
$$

Using the binomial expansion we have

$$
\alpha_{r: n}^{(a)}=\sum_{i=0}^{n-r} d_{i}(n, r) \theta(r+i) \sum_{j=0}^{\infty}(-1)^{j}\left(\begin{array}{c}
\theta(r+i)-1 \\
j
\end{array}\right) \int_{0}^{\infty} x^{a+1} e^{-(j+1) x}(x+1)^{j} d x .
$$

Thus,

$$
\begin{aligned}
\alpha_{r: n}^{(a)}= & \sum_{i=0}^{n-r} \sum_{j=0}^{\infty} \sum_{k=0}^{j}(-1)^{j} d_{i}(n, r) \theta(r+i) \\
& \times\left(\begin{array}{c}
\theta(r+i)-1 \\
j
\end{array}\right)\left(\begin{array}{l}
j \\
k
\end{array}\right) \int_{0}^{\infty} x^{a+k+1} e^{-(j+1) x} d x \\
= & \sum_{i=0}^{n-r} \sum_{j=0}^{\infty} \sum_{k=0}^{j}(-1)^{j} d_{i}(n, r) \theta(r+i)\left(\begin{array}{c}
\theta(r+i)-1 \\
j
\end{array}\right)\left(\begin{array}{l}
j \\
k
\end{array}\right) \frac{\Gamma(a+k+2)}{(j+1)^{a+k+2}} .
\end{aligned}
$$

Then we get the result (8) by using (6), hence the theorem is proved. If $\theta$ is a positive integer number, then the relation (8) becomes

$$
\begin{aligned}
\alpha_{r: n}^{(a)}= & \theta C_{r: n} \sum_{i=0}^{n-r} \sum_{j=0}^{\theta(r+i)-1} \sum_{k=0}^{j}(-1)^{i+j}\left(\begin{array}{c}
n-r \\
i
\end{array}\right)\left(\begin{array}{c}
\theta(r+i)-1 \\
j
\end{array}\right)\left(\begin{array}{l}
j \\
k
\end{array}\right) \\
& \times \frac{\Gamma(a+k+2)}{(j+1)^{a+k+2}}, \quad a=0,1,2, \ldots
\end{aligned}
$$

The single moments of order statistics from gamma distribution $G(2,1)$ can be obtained from (9) by setting $\theta=1$. 
Table 1. Values of $\beta_{1}$ and $\beta_{2}$ of $X_{r: n}$ for different values of $\theta$

\begin{tabular}{|c|c|c|c|c|c|c|c|c|c|}
\hline \multirow{2}{*}{$n$} & \multirow{2}{*}{$r$} & \multicolumn{2}{|c|}{$\theta=0.1$} & \multicolumn{2}{|c|}{$\theta=0.5$} & \multicolumn{2}{|c|}{$\theta=1.5$} & \multicolumn{2}{|c|}{$\theta=3$} \\
\hline & & $\boldsymbol{\beta}_{1}$ & $\boldsymbol{\beta}_{2}$ & $\boldsymbol{\beta}_{1}$ & $\boldsymbol{\beta}_{2}$ & $\boldsymbol{\beta}_{1}$ & $\boldsymbol{\beta}_{2}$ & $\boldsymbol{\beta}_{1}$ & $\boldsymbol{\beta}_{2}$ \\
\hline \multirow[t]{2}{*}{2} & 1 & 6.01152 & 55.7806 & 1.80963 & 7.77588 & 1.04913 & 4.66801 & 0.83161 & 4.14982 \\
\hline & 2 & 2.61306 & 12.4684 & 1.41421 & 6.00000 & 1.13866 & 5.16830 & 1.07670 & 5.03505 \\
\hline \multirow[t]{4}{*}{4} & 1 & 13.3702 & 266.712 & 1.77428 & 7.71715 & 0.85373 & 4.02074 & 0.58068 & 3.51307 \\
\hline & 2 & 5.10736 & 41.7955 & 1.35670 & 5.70018 & 0.77613 & 3.95516 & 0.63447 & 3.70419 \\
\hline & 3 & 2.87074 & 15.0541 & 1.16317 & 5.03569 & 0.83087 & 4.17702 & 0.75479 & 4.04373 \\
\hline & 4 & 1.93098 & 8.27580 & 1.20736 & 5.34612 & 1.07659 & 5.03569 & 1.05490 & 5.00999 \\
\hline \multirow[t]{6}{*}{6} & 1 & 32.3889 & 1447.16 & 1.72844 & 7.39703 & 0.75907 & 3.74508 & 0.46170 & 3.28325 \\
\hline & 2 & 9.90026 & 143.107 & 1.34822 & 5.70410 & 0.65718 & 3.64917 & 0.48760 & 3.40164 \\
\hline & 3 & 4.66034 & 35.0048 & 1.13630 & 4.87955 & 0.64666 & 3.67537 & 0.53458 & 3.51097 \\
\hline & 4 & 2.86780 & 15.3364 & 1.00628 & 4.52655 & 0.68067 & 3.79044 & 0.60683 & 3.67894 \\
\hline & 5 & 2.09612 & 9.46421 & 0.98248 & 4.52497 & 0.78035 & 4.08620 & 0.73838 & 4.02201 \\
\hline & 6 & 1.66034 & 6.98011 & 1.13866 & 5.16830 & 1.06063 & 5.01274 & 1.05203 & 5.01759 \\
\hline
\end{tabular}

The measures of skewness and kurtosis of the distribution of the $r$ th order statistic can be evaluated from the following expressions:

$$
\beta_{1}=\frac{\alpha_{r: n}^{(3)}-3 \alpha_{r: n} \alpha_{r: n}^{(2)}+2 \alpha_{r: n}^{3}}{\left(\alpha_{r: n}^{(2)}-\alpha_{r: n}^{2}\right)^{\frac{3}{2}}}
$$

and

$$
\beta_{2}=\frac{\alpha_{r: n}^{(4)}-4 \alpha_{r: n} \alpha_{r: n}^{(3)}+6 \alpha_{r: n}^{2} \alpha_{r: n}^{(2)}-3 \alpha_{r: n}^{4}}{\left(\alpha_{r: n}^{(2)}-\alpha_{r: n}^{2}\right)^{2}} .
$$

Table 1 presents the values of these measures for different choices of $r, n$ and $\theta$.

In Table 1, we present the values of $\beta_{1}$ and $\beta_{2}$ for the cases in which $n=2,4,6$ and $\theta=0.1,0.5,1.5,3$ with $1 \leqslant r \leqslant n$. For fixed $r$ and $n$, the value of $\beta_{1}$ decreases as $\theta$ increases. For fixed and $\theta \geqslant 0.5$ the value of $\beta_{2}$ increases as $r$ increases and for fixed $n$ and $\theta \leqslant 0.5$ the value of $\beta_{2}$ decreases as $r$ increases. Also, when $\theta \leqslant 0.5$ for fixed $r$ and $n$, the value of $\beta_{2}$ decreases as $\theta$ increases.

\subsection{Double Moments}

The joint p.d.f. of $X_{r: n}$ and $X_{s: n}(1 \leqslant r<s \leqslant n)$ is given by (see David, 1981)

$$
\begin{aligned}
f_{r, s: n}(x, y)= & C_{r, s: n}\{F(x ; \theta)\}^{r-1}\{F(y ; \theta)-F(x ; \theta)\}^{s-r-1}\{1-F(y ; \theta)\}^{n-s} \\
& \times f(x ; \theta) f(y ; \theta), \quad y>x>0,
\end{aligned}
$$


where $f(\cdot)$ and $F(\cdot)$ are given by (1) and (2), respectively, and

$$
C_{r, s: n}=\frac{n !}{(r-1) !(s-r-1) !(n-s) !} .
$$

$f_{r, s: n}(x, y)$ can be written as

$$
\begin{aligned}
f_{r, s: n}(x, y)= & C_{r, s: n} \sum_{i=0}^{n-s} \sum_{j=0}^{s-r-1}(-1)^{i+j}\left(\begin{array}{c}
n-s \\
i
\end{array}\right)\left(\begin{array}{c}
s-r-1 \\
j
\end{array}\right) \\
& \times\{F(x ; \theta)\}^{r+j-1}\{F(y ; \theta)\}^{s-r-1-j+i} f(x ; \theta) f(y ; \theta) .
\end{aligned}
$$

The double moments of $X_{r: n}^{a}$ and $X_{s: n}^{b} E\left(X_{r: n}^{a} X_{s: n}^{b}\right)$, denoted by $\alpha_{r, s: n}^{(a, b)}$, $1 \leqslant r<s \leqslant n$ and $a, b=0,1,2, \ldots$ is given by

$$
\alpha_{r, s: n}^{(a, b)}=\int_{0}^{\infty} \int_{x}^{\infty} x^{a} y^{b} f_{r, s: n}(x, y) d y d x .
$$

The exact explicit expression for the double moments of order statistics from the EG distribution is given by the following theorem:

Theorem 2 For $1 \leqslant r<s \leqslant n, a, b \geqslant 0$ and $\theta$ is a real value, then

$$
\begin{aligned}
\alpha_{r, s: n}^{(a, b)}= & C_{r, s: n} \theta^{2} \sum_{i=0}^{n-s} \sum_{j=0}^{s-r-1} \sum_{k=0}^{\infty} \sum_{l=0}^{k} \sum_{u=0}^{b+l+1} \sum_{v=0}^{\infty} \sum_{p=0}^{v}(-1)^{i+j+k+v}\left(\begin{array}{c}
n-s \\
i
\end{array}\right) \\
& \times\left(\begin{array}{c}
s-r-1 \\
j
\end{array}\right)\left(\begin{array}{c}
\theta(s-r-j+i)-1 \\
k
\end{array}\right)\left(\begin{array}{c}
k \\
l
\end{array}\right)\left(\begin{array}{c}
\theta(r+j)-1 \\
v
\end{array}\right) \\
& \times\left(\begin{array}{c}
v \\
p
\end{array}\right) \frac{\Gamma(b+l+2) \Gamma(a+u+p+2)}{u !(k+1)^{b+l-u+2}(k+v+2)^{a+u+p+2}} .
\end{aligned}
$$

Proof. From (1), (2) and (12) we get

$$
\begin{aligned}
\alpha_{r, s: n}^{(a, b)}= & \theta^{2} C_{r, s: n} \sum_{i=0}^{n-s} \sum_{j=0}^{s-r-1}(-1)^{i+j}\left(\begin{array}{c}
n-s \\
i
\end{array}\right)\left(\begin{array}{c}
s-r-1 \\
j
\end{array}\right) \int_{0}^{\infty} \int_{x}^{\infty} x^{a+1} y^{b+1} e^{-x} e^{-y} \\
& \times\left\{1-e^{-x}(x+1)\right\}^{\theta(r+j)-1}\left\{1-e^{-y}(y+1)\right\}^{\theta(s-r-j+i)-1} d y d x
\end{aligned}
$$




$$
\begin{aligned}
= & \theta^{2} C_{r, s: n} \sum_{i=0}^{n-s} \sum_{j=0}^{s-r-1}(-1)^{i+j}\left(\begin{array}{c}
n-s \\
i
\end{array}\right)\left(\begin{array}{c}
s-r-1 \\
j
\end{array}\right) \\
& \int_{0}^{\infty} x^{a+1} e^{-x}\left\{1-e^{-x}(x+1)\right\}^{\theta(r+j)-1} I_{1}(x) d x
\end{aligned}
$$

where

$$
\begin{aligned}
I_{1}(x) & =\int_{x}^{\infty} y^{b+1} e^{-y}\left[1-e^{-y}(y+1)\right]^{\theta(s-r-j+i)-1} d y \\
& =\sum_{k=0}^{\infty} \sum_{l=0}^{k}(-1)^{k}\left(\begin{array}{c}
\theta(s-r-j+i)-1 \\
k
\end{array}\right)\left(\begin{array}{l}
k \\
l
\end{array}\right) \\
& \sum_{u=0}^{b+l+1} \frac{x^{u} e^{-(k+1) x}}{(k+1)^{b+l+2-u}} \cdot \frac{\Gamma(b+l+2)}{u !} .
\end{aligned}
$$

Substituting $I_{1}(x)$ in (14) we get

$$
\begin{aligned}
\alpha_{r, s: n}^{(a, b)}= & \theta^{2} C_{r, s: n} \sum_{i=0}^{n-s} \sum_{j=0}^{s-r-1} \sum_{k=0}^{\infty} \sum_{l=0}^{k} \sum_{u=0}^{b+l+1}(-1)^{i+j+k}\left(\begin{array}{c}
n-s \\
i
\end{array}\right) \\
& \times\left(\begin{array}{c}
s-r-1 \\
j
\end{array}\right)\left(\begin{array}{c}
\theta(s-r-j+i)-1 \\
k
\end{array}\right)\left(\begin{array}{c}
k \\
l
\end{array}\right) \\
& \times \frac{\Gamma(b+l+2)}{u !(k+1)^{b+l+2-u}} \int_{0}^{\infty} x^{a+u+1} e^{-(k+2) x}\left\{1-e^{-x}(x+1)\right\}^{\theta(r+j)-1} d x \\
= & \theta^{2} C_{r, s: n} \sum_{i=0}^{n-s} \sum_{j=0}^{s-r-1} \sum_{k=0}^{\infty} \sum_{l=0}^{k} \sum_{u=0}^{b+l+1} \sum_{v=0}^{\infty} \sum_{p=0}^{v}(-1)^{i+j+k+v} \\
& \times\left(\begin{array}{c}
n-s \\
i
\end{array}\right)\left(\begin{array}{c}
s-r-1 \\
j
\end{array}\right)\left(\begin{array}{c}
\theta(s-r-j+i)-1 \\
k \\
l
\end{array}\right) \\
& \times\left(\begin{array}{c}
\theta(r+j)-1 \\
v
\end{array}\right)
\end{aligned}
$$

The integral in the last equation is a gamma function, hence the theorem 
is proved.

If $\theta$ is a positive integer number, then the relation (13) becomes

$$
\begin{aligned}
\alpha_{r, s: n}^{(a, b)}= & C_{r, s: n} \theta^{2} \sum_{i=0}^{n-s} \sum_{j=0}^{s-r-1} \sum_{k=0}^{\theta(s-r-j+i)-1} \sum_{l=0}^{k} \sum_{u=0}^{b+l+1} \sum_{v=0}^{\theta(r+j)-1} \sum_{p=0}^{v}(-1)^{i+j+k+v} \\
& \times\left(\begin{array}{c}
n-s \\
i
\end{array}\right)\left(\begin{array}{c}
s-r-1 \\
j
\end{array}\right)\left(\begin{array}{c}
\theta(s-r-j+i)-1 \\
k
\end{array}\right)\left(\begin{array}{c}
k \\
l
\end{array}\right)\left(\begin{array}{c}
\theta(r+j)-1 \\
v
\end{array}\right)\left(\begin{array}{l}
v \\
p
\end{array}\right) \\
& \times \frac{\Gamma(b+l+2) \Gamma(a+u+p+2)}{u !(k+1)^{b+l-u+2}(k+v+2)^{a+u+p+2}}, \quad a, b=0,1,2, \ldots
\end{aligned}
$$

The double moments of order statistics from gamma distribution $G(2,1)$ can be obtained from (16) by setting $\theta=1$.

\section{Recurrence Relations Between Moments of Or- der Statistics}

With $f(\cdot)$ and $F(\cdot)$ are given in (1) and (2), respectively. Then by making use of the characterization differential equation in (3), we establish in this section recurrence relations for the single and double moments of order statistics as follows:

\subsection{Recurrence Relations for Single Moments}

Theorem 3 For $1<r \leqslant n-1, a \geqslant 0$ and $L=\frac{1}{\theta}=1,2,3, \ldots, r-1$

$$
\begin{aligned}
\alpha_{r: n}^{(a+1)}+\alpha_{r: n}^{(a)}= & \frac{n !(r-L) !}{L(a+2)(r-1) !(n-L) !}\left\{\alpha_{r-L+1: n-L}^{(a+2)}-\alpha_{r-L: n-L}^{(a+2)}\right\} \\
& +\frac{r}{L(a+2)}\left\{\alpha_{r: n}^{(a+2)}-\alpha_{r+1: n}^{(a+2)}\right\} .
\end{aligned}
$$

Proof. From (3) and (4), we get

$$
E\left(X_{r: n}^{a+1}+X_{r: n}^{a}\right)=\int_{0}^{\infty}\left(x^{a+1}+x^{a}\right) f_{r: n}(x) d x,
$$




$$
\begin{aligned}
E\left(X_{r: n}^{a+1}+X_{r: n}^{a}\right)= & C_{r: n} \int_{0}^{\infty} x^{a}(x+1)\{F(x)\}^{r-1}\{1-F(x)\}^{n-r} f(x) d x \\
= & C_{r: n} \theta\left[\int_{0}^{\infty} x^{a+1}\{F(x)\}^{r-L}\{1-F(x)\}^{n-r} d x\right. \\
& \left.-\int_{0}^{\infty} x^{a+1}\{F(x)\}^{r}\{1-F(x)\}^{n-r} d x\right] .
\end{aligned}
$$

Integrating by parts, we get the result, hence the theorem is proved.

For $\theta=1,1<r \leqslant n-1, a \geqslant 0$, and by using the identity (see Arnold et al., 1992),

$$
i \mu_{i+1: n}^{(m)}+(n-i) \mu_{i: n}^{(m)}=n \mu_{i: n-1}^{(m)}
$$

the recurrence relation in (17) is valid for gamma distribution $G(2,1)$, which takes the form

$$
\alpha_{r: n}^{(a+1)}+\alpha_{r: n}^{(a)}=\frac{n-r+1}{a+2}\left\{\alpha_{r: n}^{(a+2)}-\alpha_{r-1: n}^{(a+2)}\right\}
$$

which is a special case when $m=2$ from the recurrence relation

$$
\mu_{r: n}^{(k)}=\mu_{r-1: n-1}^{(k)}+\frac{k}{n} \sum_{t=0}^{m-1} \frac{\mu_{r: n}^{(t+k-m)}}{t !},
$$

that was derived by Joshi (1979).

\subsection{Recurrence Relations for Double Moments}

Theorem 4 For $L+1<r+1<s \leqslant n, a, b \geqslant 0$ and $L=\frac{1}{\theta}=1,2,3, \ldots, r-1$

$$
\begin{aligned}
\alpha_{r, s: n}^{(a+1, b)}+\alpha_{r, s: n}^{(a, b)}= & \frac{n !(r-L) !}{L(a+2)(r-1) !(n-L) !}\left\{\alpha_{r-L+1, s-L: n-L}^{(a+2, b)}-\alpha_{r-L, s-L: n-L}^{(a+2, b)}\right\} \\
& +\frac{r}{L(a+2)}\left\{\alpha_{r, s: n}^{(a+2, b)}-\alpha_{r+1, s: n}^{(a+2, b)}\right\}
\end{aligned}
$$


Proof. From (3) and (10), we obtain

$$
\begin{aligned}
E\left(X_{r, s: n}^{a+1} Y_{r, s: n}^{b}+X_{r, s: n}^{a} Y_{r, s: n}^{b}\right) & =\int_{0}^{\infty} \int_{0}^{y}\left(x^{a+1} y^{b}+x^{a} y^{b}\right) f_{r, s: n}(x, y) d x d y \\
& =C_{r, s: n} \int_{0}^{\infty} y^{b}\{1-F(y)\}^{n-s} I(y) f(y) d y,
\end{aligned}
$$

where

$$
\begin{aligned}
I(y)= & \int_{0}^{y} x^{a}(x+1)\{F(x)\}^{r-1}\{F(y)-F(x)\}^{s-r-1} f(x) d x \\
= & \theta\left[\int_{0}^{y} x^{a+1}\{F(x)\}^{r-L}\{F(y)-F(x)\}^{s-r-1} d x\right. \\
& \left.-\int_{0}^{y} x^{a+1}\{F(x)\}^{r}\{F(y)-F(x)\}^{s-r-1} d x\right] \\
= & \frac{\theta}{a+2}\left[(s-r-1) \int_{0}^{y} x^{a+2}\{F(x)\}^{r-L}\{F(y)-F(x)\}^{s-r-2} f(x) d x\right. \\
& -(r-L) \int_{0}^{y} x^{a+2}\{F(x)\}^{r-L-1}\{F(y)-F(x)\}^{s-r-1} f(x) d x \\
& -(s-r-1) \int_{0}^{y} x^{a+2}\{F(x)\}^{r}\{F(y)-F(x)\}^{s-r-2} f(x) d x \\
& \left.+r \int_{0}^{y} x^{a+2}\{F(x)\}^{r-1}\{F(y)-F(x)\}^{s-r-1} f(x) d x\right] .
\end{aligned}
$$

Integrating by parts and substituting the above expression of $I(y)$ in (19), then simplifying the resulting equation, we obtain (18). Hence the theorem is proved. 
For $\theta=1,2<r+1<s \leqslant n$, and $a, b \geqslant 0$, the recurrence relation in (18) is valid for gamma distribution $G(2,1)$, which takes the form

$\alpha_{r, s: n}^{(a+1, b)}+\alpha_{r, s: n}^{(a, b)}=\frac{n}{a+2}\left\{\alpha_{r, s-1: n-1}^{(a+2, b)}-\alpha_{r-1, s-1: n-1}^{(a+2, b)}\right\}+\frac{r}{a+2}\left\{\alpha_{r, s: n}^{(a+2, b)}-\alpha_{r+1, s: n}^{(a+2, b)}\right\}$.

\section{MGF of Order Statistics}

Let $X_{1: n} \leqslant X_{2: n} \leqslant \cdots \leqslant X_{n: n}$ be the order statistics from the EG distribution given in (1). Then MGF for the single moments of order statistics are given as follows:

\subsection{MGF for Single Moments}

The MGF of the $r$ th order statistics $X_{r: n}$ denoted by $M_{r: n}(t)$ is given (see David, 1981, and Arnold et al., 1992) by

$$
M_{r: n}(t)=E\left(e^{t X_{r: n}}\right)=\int_{0}^{\infty} e^{t x} f_{r: n}(x) d x
$$

where $f_{r: n}(x)$ is defined in (4).

The exact explicit expression for the MGF for single moments of order statistics from EG distribution is given by the following theorem:

Theorem 5 For $1 \leqslant r \leqslant n$ and $\theta$ is a real value, then

$$
M_{r: n}(t)=\theta C_{r: n} \sum_{i=0}^{n-r} \sum_{j=0}^{\infty} \sum_{k=0}^{j}(-1)^{i+j}\left(\begin{array}{c}
n-r \\
i
\end{array}\right)\left(\begin{array}{c}
\theta(r+i)-1 \\
j
\end{array}\right)\left(\begin{array}{l}
j \\
k
\end{array}\right) \frac{\Gamma(k+2)}{(1-t+j)^{k+2}}
$$

Proof. From (1), (5) and (22) we get

$$
M_{r: n}(t)=\theta \sum_{i=0}^{n-r} d_{i}(n, r)(r+i) \int_{0}^{\infty} x e^{-(1-t) x}\left\{1-e^{-x}(x+1)\right\}^{\theta(r+i)-1} d x .
$$


Using the binomial expansion we have

$$
\begin{aligned}
M_{r: n}(t) & =\theta \sum_{i=0}^{n-r} d_{i}(n, r)(r+i) \sum_{j=0}^{\infty}(-1)^{j}\left(\begin{array}{c}
\theta(r+i)-1 \\
j
\end{array}\right) \int_{0}^{\infty} x e^{-(1-t+j) x}(x+1)^{j} d x \\
& =\theta \sum_{i=0}^{n-r} \sum_{j=0}^{\infty} \sum_{k=0}^{j}(-1)^{j} d_{i}(n, r)(r+i)\left(\begin{array}{c}
\theta(r+i)-1 \\
j
\end{array}\right)\left(\begin{array}{l}
j \\
k
\end{array}\right) \frac{\Gamma(k+2)}{(1-t+j)^{k+2}} .
\end{aligned}
$$

Then we get the result (23) by using (6), hence the theorem is proved. If $\theta$ is a positive integer number, then the relation (23) becomes

$$
\begin{aligned}
M_{r: n}(t)= & \theta C_{r: n} \sum_{i=0}^{n-r} \sum_{j=0}^{\theta(r+i)-1} \sum_{k=0}^{j}(-1)^{i+j}\left(\begin{array}{c}
n-r \\
i
\end{array}\right)\left(\begin{array}{c}
\theta(r+i)-1 \\
j
\end{array}\right) \\
& \times\left(\begin{array}{l}
j \\
k
\end{array}\right) \frac{\Gamma(k+2)}{(1-t+j)^{k+2}} .
\end{aligned}
$$

The MGF for single moments of order statistics from gamma distribution $G(2,1)$ can be obtained from (24) by setting $\theta=1$.

\subsection{Recurrence Relations for MGF}

We establish a recurrence relation for the MGF for single moments of order statistics by making use of the following characterization differential equation:

$$
\left.\theta F(x ; \theta)=\left\{x^{-1}\left(e^{x}-1\right)-1\right\} f(x ; \theta)\right\},
$$

the MGF of $X$ is denoted by $M_{X}(t)$, which its $i$ th derivative is $M_{X}^{(i)}(t)$.

The following theorem gives the recurrence relations for the MGF of order statistics.

Theorem 6 For $1 \leqslant r \leqslant n-1$, then

$$
\begin{aligned}
M_{r+1: n}(t)-t M_{r+1: n}^{\prime}(t) & =-\frac{t^{2}}{r \theta} M_{r: n}(t+1)+\left(\frac{t^{2}}{r \theta}+1\right) M_{r: n}(t) \\
& +\left(\frac{t^{2}}{r \theta}-t\right) M_{r: n}^{\prime}(t) .
\end{aligned}
$$


(ii)

$$
\begin{aligned}
-(i-1) M_{r+1: n}^{(i)}(t)-t M_{r+1: n}^{(i+1)}(t)= & \frac{-t^{2}}{r \theta} M_{r: n}^{(i)}(t+1)+i\left(\frac{-2 t}{r \theta}\right) M_{r: n}^{(i-1)}(t+1) \\
& +\left(\begin{array}{c}
i \\
2
\end{array}\right)\left(\frac{-2}{r \theta}\right) M_{r: n}^{(i-2)}(t+1)+\left\{\frac{t^{2}}{r \theta}+\frac{2 i t}{r \theta}\right. \\
& +(1-i)\} M_{r: n}^{(i)}(t)+\left(\frac{2}{r \theta}\right)\{i t \\
& \left.+\left(\begin{array}{c}
i \\
2
\end{array}\right)\right\} M_{r: n}^{(i-1)}(t) \\
& +\left(\begin{array}{c}
i \\
2
\end{array}\right)\left(\frac{2}{r \theta}\right) M_{r: n}^{(i-2)}(t) \\
& +\left(\frac{t^{2}}{r \theta}-t\right) M_{r: n}^{(i+1)}(t), \quad i \geqslant 2, \quad(27)
\end{aligned}
$$

and consequently,

$$
\alpha_{r+1: n}^{(2)}=\frac{2}{r \theta} \sum_{j=3}^{\infty} \frac{1}{j !} \alpha_{r: n}^{(j)}+\left(1+\frac{1}{r \theta}\right) \alpha_{r: n}^{(2)}
$$

(iv) $\quad(i-1) \alpha_{r+1: n}^{(i)}=\left(\begin{array}{c}i \\ 2\end{array}\right)\left(\frac{2}{r \theta}\right) \sum_{j=2}^{\infty} \frac{1}{j !} \alpha_{r: n}^{(i-2+j)}+(i-1) \alpha_{r: n}^{(i)}, \quad i \geqslant 2$.

Proof. It is clear that

$$
\begin{aligned}
M_{r: n}(t+1)= & C_{r: n} \int_{0}^{\infty} e^{(t+1) x}\{F(x ; \theta)\}^{r-1}\{1-F(x ; \theta)\}^{n-r} f(x ; \theta) d x \\
= & C_{r: n} \int_{0}^{\infty} x e^{t x}\left(\frac{e^{x}-1}{x}-1\right)\{F(x ; \theta)\}^{r-1}\{1-F(x ; \theta)\}^{n-r} \\
& \times f(x ; \theta) d x+M_{r: n}^{\prime}(t)+M_{r: n}(t) .
\end{aligned}
$$

From (25) and (30), we get

$M_{r: n}(t+1)=C_{r: n} \theta \int_{0}^{\infty} x e^{t x}\{F(x ; \theta)\}^{r-1+1}\{1-F(x ; \theta)\}^{n-r} d x+M_{r: n}^{\prime}(t)+M_{r: n}(t)$. 
Integrating by parts, we obtain

$$
\begin{aligned}
M_{r: n}(t+1)= & \frac{d}{d t} C_{r: n} \theta\left\{\frac{n-r}{t} \int_{0}^{\infty} e^{t x}\{F(x ; \theta)\}^{r}\{1-F(x ; \theta)\}^{n-r-1} f(x ; \theta) d x\right. \\
& \left.+\frac{-r}{t} \int_{0}^{\infty} e^{t x}\{F(x ; \theta)\}^{r-1}\{1-F(x ; \theta)\}^{n-r} f(x ; \theta) d x\right\} \\
& +M_{r: n}^{\prime}(t)+M_{r: n}(t) .
\end{aligned}
$$

Simplifying, we get (26).

Differentiating equation (26) with respect to $t$, we have

$$
\begin{aligned}
M_{r+1: n}^{\prime}(t)-t M_{r+1: n}^{\prime \prime}(t)-M_{r+1: n}^{\prime}(t)= & \frac{-t^{2}}{r \theta} M_{r: n}^{\prime}(t+1)-\frac{2 t}{r \theta} M_{r: n}(t+1) \\
& +\left(\frac{t^{2}}{r \theta}+\frac{2 t}{r \theta}\right) M_{r: n}^{\prime}(t)+\frac{2 t}{r \theta} M_{r: n}(t) \\
& +\left(\frac{t^{2}}{r \theta}-t\right) M_{r: n}^{\prime \prime}(t) .
\end{aligned}
$$

Setting $t=0$ in (32), we get

$$
-\alpha_{r+1: n}^{(2)}=\frac{-2}{r \theta} M_{r: n}(1)+\frac{2}{r \theta}-\alpha_{r: n}^{(2)}+\frac{2}{r \theta} \alpha_{r: n} .
$$

After some simplification, we get (28). Also by differentiating equation (26), with respect to $t, i$ times, we get (27). Setting $t=0$ in (27), we get (29). Thus the theorem is proved.

\section{Applications}

The results established in this paper and some similar generalizations can be used to determine the mean, variance and the coefficients of skewness and kurtosis. The moments can also be used for finding best linear unbiased estimators (BLUE's) of location and scale parameters and conditional moments. Some of the results are then used to characterize the distribution. Shawky and Bakoban (2009) has discussed order statistics from EG distribution and associated inference. Based on the moments of order statistics, the BLUE's of the location and scale parameters of EG distribution under Type-II censoring were obtained. The variances and covariances of these estimators were 
also presented.

\section{Acknowledgments}

The authors express their sincere thanks to the editor and the referees for their constructive criticisms and excellent suggestions which led to a considerable improvement in the presentation of the paper.

\section{References}

Barz, G., Johnson, B. (1998). Modelling the Prices of Commodities that are Costly to Store: The Case of Electricity, Proceedings of the Chicago Risk Management Conference, Chicago, IL.

Arnold, B.C., Balakrishnan, N. and Nagaraja, H.N. (1992). A First Course in Order Statistics. Wiley, New York.

Balakrishnan, N. and Aggarwala, R. (1998). Recurrence relations for single and product moments of order statistics from a generalized logistic distribution with applications to inference and generalizations to double truncation. In: Balakrishnan, N. and Rao, C. R. (Eds.), Handbook of Statistics, Vol. 17, Elsevier Science, Amsterdam, 85- 126.

Balakrishnan, N. and Cohen, A.C. (1991). Order Statistics and Inference: Estimation Methods. Academic Press, San Diego.

Balakrishnan, N. and Sultan, K.S. (1998). Recurrence relations and identities for moments of order statistics. In: Balakrishnan, N. and Rao, C. R. (Eds.), Handbook of Statistics, Vol. 16, Elsevier Science, Amsterdam, 149- 228.

Balasubramanian, K. and Beg, M.I. (2004). Identities in order statistics-from moments to arbitrary distributions. Statistics, 38, 43-48.

Basu, A.P. and Singh, B. (1998). Order statistics in exponential distribution. In: Balakrishnan, N. and Rao, C. R. (Eds.), Handbook of Statistics, Vol. 17, Elsevier Science, Amsterdam, $3-23$.

Breiter, M.C. and Krishnaiah, P.R. (1968). Tables for the moments of gamma order statistics. Sankhyā The Indian Journal of Statistics, B 30, 59-72.

David, H.A. (1981). Order Statistics. Second Edition, Wiley, New York.

David, H.A. (1995). On recurrence relations for order statistics. Statistics and Probability Letters, 24, 133-138.

David, H.A. and Nagaraja, H.N. (2003). Order Statistics. Third Edition, Wiley, New Jersey. 
Gupta, S.S. (1960). Order statistics from the gamma distribution. Technometrics, 2, 243-262.

Joshi, P.C. (1979). On the moments of gamma order statistics. Naval Research Logistic Quarterly, 26, 675-679.

Krishnaiah, P.R. and Rizvi, M.H. (1967). A note on moments of gamma order statistics. Technometrics, 9, 315-318.

Raqab, M.Z. (1998). Order statistics from the Burr type X model. Computers and Mathematics with Applications, 36, 111-120.

Raqab, M.Z. (2004). Generalized exponential distribution: Moments of order statistics. Statistics, 38, 29-41.

Shawky, A.I. and Bakoban, R.A. (2008a). Bayesian and non-Bayesian estimations on the exponentiated gamma distribution. Applied Mathematical Sciences, 5, 2521-2530.

Shawky, A.I. and Bakoban, R.A. (2008b). Characterization from exponentiated gamma distribution based on record values. Journal of Statistical Theory and Applications, 7, 263-278.

Shawky, A.I. and Bakoban, R.A. (2008c). Certain characterizations of the exponentiated gamma distribution. Accepted for publication in Journal of Approximation Theory and Applications.

Shawky, A.I. and Bakoban, R.A. (2009). Order statistics from exponentiated gamma distribution and associated inference. The International Journal of Contemporary Mathematical Sciences, 4,71-91.

Young, D.H. (1971). Moment relations for order statistics of the standardized gamma distribution and the inverse multinomial distribution. Biometrika, 58, 637-640.

\author{
A. I. Shawky \\ Department of Statistics, \\ Faculty of Sciences, \\ King Abdulaziz University, \\ Jeddah, Saudi Arabia.
}

\author{
R. A. Bakonan \\ Department of Mathematics, \\ Scientific Section, \\ Girls College of Education, \\ King Abdulaziz University, \\ Jeddah, Saudi Arabia. \\ email: rbaboban@yahoo.com
}

